\title{
BMJ Open Differences in the outcomes of adjuvant chemotherapy for colon cancer prescribed by physicians in different disciplines: a population-based study in Taiwan
}

Cheng-I Hsieh, ${ }^{1,2,3}$ Raymond Nien-Chen Kuo, ${ }^{3,4}$ Chun-Chieh Liang, ${ }^{3}$ Hsin-Yun Tsai, ${ }^{5}$ Kuo-Piao Chung ${ }^{3,4}$

To cite: Hsieh C-I, Kuo RN-C, Liang C-C, et al. Differences in the outcomes of adjuvant chemotherapy for colon cancer prescribed by physicians in different disciplines: a population-based study in Taiwan. BMJ Open 2018;8:e021341. doi:10.1136/ bmjopen-2017-021341

- Prepublication history for this paper is available online. To view these files, please visit the journal online (http://dx.doi. org/10.1136/bmjopen-2017021341).

Received 27 December 2017 Revised 28 0ctober 2018 Accepted 2 November 2018

Check for updates

(C) Author(s) (or their employer(s)) 2018. Re-use permitted under CC BY-NC. No commercial re-use. See rights and permissions. Published by BMJ.

For numbered affiliations see end of article.

Correspondence to Dr Raymond Nien-Chen Kuo; nckuo@ntu.edu.tw

\section{ABSTRACT}

Objectives One feature unique to the Taiwanese healthcare system is the ability of physicians other than oncologists to prescribe systemic chemotherapy. This study investigated whether the care paths implemented by oncologists and non-oncologists differ with regard to patient outcomes.

Setting Data from the Taiwan Cancer Registry and National Health Insurance Database were linked to identify patients with colon cancer who underwent colectomy as first treatment within 3 months of diagnosis and adjuvant chemotherapy between 2005 and 2009.

Participants and methods Postoperative patients who underwent adjuvant chemotherapy were included in this study. The exclusion criteria included patients with stage IV disease, a positive surgical margin and early disease recurrence. Among the patients presenting with multiple primary cancers, we also excluded patients who were diagnosed with colon cancer but for whom this was not the first primary cancer. The variables included sex, age, comorbidities, disease stage, chemotherapy cycle and changes in treatment regimen as well as the specialty of treatment providers and their case volume. Cox regression models and Kaplan-Meier analysis were used to examine differences in outcomes in the matched cohorts.

Results We examined 3534 patients who were prescribed adjuvant chemotherapy by physicians from different disciplines. In terms of 5-year diseasefree survival, no significant difference was observed between the groups of oncologists or surgeons among patients with stage II $(90.02 \%$ vs $88.99 \%)$ or stage III (77.64\%vs79.99\%) diseases. Patients who were subjected to changes in their chemotherapy regimens presented recurrence rates higher than those who were not.

Conclusions The discipline of practitioners is seldom taken into account in most series. This is the first study to provide empirical evidence demonstrating that the outcomes of patients with colon cancer do not depend on the treatment path, as long as the selection criteria for adjuvant chemotherapy is appropriate. Further study will be required before making any further conclusions.
Strengths and limitations of this study

- This is the first study to examine the differential outcomes of patients with cancer under different care paths, which are of particular importance in countries where adjuvant chemotherapy prescribed by physicians from different disciplines is the norm.

- We excluded many ineligible patients based on strict analysis criteria and used propensity score matching to reduce the bias of confounding factors between two groups.

- The study design links two large national datasets covering a large number of patients with records related to long-term follow-up.

- The primary limitation of this study was our inability to obtain data pertaining to variations in dose intensity across providers and clinical information of patients.

\section{INTRODUCTION}

Shared care refers to the joint participation of physicians in the planning of patient care. This approach has been shown to improve cancer outcomes by helping to coordinate care to ensure the timely administration of adjuvant chemotherapy and extend survival. ${ }^{12}$ Innovations in healthcare have resulted in highly specialised treatment regimes. For example, coronary artery bypass grafts performed by cardiothoracic surgeons have been replaced with percutaneous catheterisation intervention performed by cardiologists. ${ }^{3} 4$ This has led to the blurring of professional boundaries, as discussed by the American Society of Gynecologic Oncology. ${ }^{5}$ Another situation is the long-simmering conflict between breast surgeons or radiologists over who should perform an ultrasound or stereotactic biopsies. ${ }^{6}$ These disputes demonstrate the 
interprofessional boundary changes that have occurred in the healthcare workforce. ${ }^{7}$

In most western countries, physicians stay close to their areas of specialisation and rarely violate interprofessional boundaries. ${ }^{8}$ Surgeons and radiation oncologists play distinct roles in cancer treatment. Medical oncologists are a subspecialty dedicated to the 'total management' of patients with cancer and tasked with coordinating a multidisciplinary approach from initial diagnosis through cure to end-of-life care. ${ }^{9}$ Nurses prescribing medication is another example of the permeable role boundary of oncologists. ${ }^{10} 11$ The literature indicates that a reluctance on the part of surgeons to refer patients to oncologists or the disparities in receipt of adjuvant therapy has to do with the age and race of patients as well as their expressed preferences with regard to chemotherapy. ${ }^{8}{ }^{12}$ In Taiwan, chemotherapy is reimbursed irrespective of the specialty of the provider. It has been assumed that this provides a financial incentive for the horizontal substitution of surgeons in performing the tasks normally assumed by oncologists. However, differences in outcomes among patients treated by different subspecialists must be elucidated before addressing this issue.

The formidable gastrointestinal side effects of chemotherapy and neutropenic fever have been greatly alleviated through the adoption of more efficient antiemetics and granulocyte colony stimulation factor. These medical advances have improved outcomes and facilitated the administration of chemotherapy, which has in turn opened the door to practitioners in other disciplines to move into areas conventionally regarded as the 'turf' of oncologists. When neutropenia or infection is encountered after chemotherapy, the doses can be reduced or the schedule delayed; however, these changes tend to undermine tumour response due to a compromised dose intensity. Moreover, regimen changes in the form of omissions or replacement with new agents can also affect survival benefits. ${ }^{13-16}$ The aforementioned skills and knowledge all fall within the discipline of oncology. Thus, the segregation of oncologists from the multidisciplinary team approach represents a deprofessionalisation of oncologists as well as an example of poor collaboration and a threat to the quality of care.

Our objective in this study was to determine whether the care paths implemented by oncologists and non-oncologists differ with regard to patient outcomes. From a logistical perspective, two distinctive forms of in-house cancer care can be observed in Taiwan: (1) surgeons consulting with oncologists in the prescription of postoperative chemotherapy and (2) surgeons prescribing adjuvant chemotherapy and conducting follow-up. From a practical perspective, it is impossible to conduct randomised clinical trials to compare the differences in outcomes among patients following different care paths. The results of this study are of particular relevance in regions facing a shortage of oncologists and in regions where there are concerns pertaining to the outcomes of patients receiving adjuvant treatment from clinicians other than oncologists.
MATERIALS AND METHODS

\section{Study population}

The sample included patients who were first diagnosed with colon cancer according to the American Joint Committee on Cancer Stages I-III (ICD-0-3=C18) between January 2005 and December 2009. All participants had undergone colectomy, as verified by the Taiwan Cancer Registry (TCR). The data were linked to the National Health Insurance Research Database (NHIRD) for follow-up between Jan. 2005 and December 2012. Patients with pre-existing cancer and those younger than 20 years were excluded from analysis.

\section{Data sources}

This study linked population-based data collected from two databases in Taiwan: the TCR and the NHIRD. The TCR collects cancer-specific data, including cancer type, cancer stage, surgical margin and details of the surgical procedures used. The data are abstracted into a standard report form by trained cancer registrars at each hospital, before being submitted with supporting medical records and passed through a computerised logic check. From the NHIRD, we retrieved the patient ID, date of ambulatory or inpatient care, disease classification codes (ICD-9-CM codes), physician ID, physician specialty, hospital ID, procedures performed (surgical and non-surgical) and medications prescribed in each case. The two databases were linked to identify cases of cancer recurrence. The IDs of the patients, physicians and hospitals were all encrypted using the same algorithm to enable the crosslinking of data, while protecting privacy.

\section{Patient selection and variables}

Postoperative care paths were determined according to whether adjuvant chemotherapy administration and follow-up were performed by oncologists (path 1) or surgeons (path 2) until disease recurrence. The adjuvant chemotherapy regimen included either single-agent fluorouracil or in combination with or without leucovorin and oxaliplatin. Any cases of other oral chemotherapy, unconventional regimens and off-label usages were excluded.

To avoid the misclassification of adjuvant therapy, we considered only chemotherapy prescribed within a designated period. The period began after curative colectomy and ended (1) on the claims date after which no new treatment for colon cancer was received within 3 months, including surgery, chemotherapy and radiation; (2) at the time of cancer recurrence; or (3) 12 months after surgery, whichever occurred first. Differentiating salvage chemotherapy for recurrence after adjuvant chemotherapy from true upfront chemotherapy in early recurrence (within 1 year of diagnosis) can be fraught with ambiguities. Thus, we adopted a strict criterion of ineligibility for all patients presenting early recurrence, which resulted in the exclusion of 613 patients from analysis. We also excluded 235 patients who were diagnosed with colon cancer but for whom this was not the first primary cancer (in the sequence of malignant and non-malignant 
neoplasms over the lifetime of the patient). The recommendations for chemotherapy were derived from clinical trials and guidelines outlined by Roswell Park, NSABP C- $04,{ }^{1718}$ the Mayo Clinic ${ }^{1819}$ and the Mosaic regimen. ${ }^{20-22}$ A change in the regimen was defined as either the addition of a new chemotherapeutic agent or the removal of an existing agent from the original protocol.

The patient's sex, age, stage of cancer, comorbidities and history of regimen changes were used for matching and were also controlled in Cox regression models. The designation of comorbidity was based on a version of the National Cancer Institute (NCI) Comorbidity Index, in which cases are classified according to comorbidity scores (ie, 1,2 or $\geq 3$ ). ${ }^{23}$ The case volume of physicians was controlled by counting the annual number of patients newly diagnosed with colon cancer. Recurrence was defined as a metastatic or recurrent disease before or after the completion of adjuvant treatment or within the follow-up period (>12 months). Cases of recurrence were defined using diagnostic codes (ICD-9 codes: 196.0-3, $196.5-6,196.8-9,197.0-8,198.0-8,199.0-1)$ or the implementation of a new treatment modality (eg, surgery or radiotherapy) before the end of a cycle of adjuvant chemotherapy or 3 months or more after the completion of this adjuvant chemotherapy. Patients with secondary malignancies were excluded from the analysis. Diseasefree survival (DFS) was defined as the time between colectomy and disease recurrence. Billing codes were used to assign patients to the surgeon who performed the definitive surgery and prescribed systemic chemotherapy. For the oncological care path, patients were assigned to the medical oncologist who billed for most of the visits and oversaw adjuvant chemotherapy within 1 year after colectomy. The case volume of the treatment provider was defined in terms of the number of patients on which the surgeon operated or who received systemic chemotherapy or care in a given year, as determined in quartiles of case volume (ie, $<25 \%, 25 \%-50 \%, 51 \%-75 \%,>75 \%$ ).

\section{Statistical analysis}

Descriptive statistics were used to evaluate baseline characteristics. We adopted propensity score methods similar to those described in previous studies ${ }^{24} 25$ to create a cohort of matched patients (ie, sharing similar characteristics). The scores were calculated using logistic regression to estimate the probability of each patient receiving adjuvant chemotherapy on the basis of sex, age, stage, comorbidities and change in regimen. Patient cohorts on both paths were matched using a greedy-matching algorithm to formulate a 1:1 case-control match ratio using callipers with a width of $<0.2$ standard deviations of the logit of propensity scores. The degree of balance in characteristics was compared using the Mantel-Haenszel test and generalised estimating equation regression. The association between various care paths and patient DFS was examined using the Cox proportional hazards model. The results are reported as HRs in conjunction with 95\% CIs. The Kaplan-Meier method was used to estimate the
DFS of patients with stage II and stage III colon cancer. We performed a log-rank test to test the difference in DFS between the care paths of oncologists and surgeons. All statistical tests were two-sided, and a computed $\mathrm{p}$ value $<0.05$ was considered significant.

\section{Patient and public involvement}

No patients or members of the public were involved in the design or implementation of this study. Patients and the general public will be informed of the study results via peer-reviewed journals.

\section{RESULTS}

A total of 25005 patients with primary colon cancer were identified from the TCR data. Among these patients, 20678 had undergone colectomy surgery. We further limited the cohort to stage I-III, class 1 and 2 patients with a histology of adenocarcinoma who had undergone postoperative adjuvant chemotherapy (figure 1). This left a total of 3534 matched patients eligible for analysis. The proportions of men and women were $54.2 \%$ and $45.8 \%$, respectively (table 1). Among them, $50.5 \%$ of patients were older than 60 years and $23.8 \%$ were elderly patients ( $>70$ years old). Patients with stage II and III colon cancer accounted for $26.1 \%$ and $72.5 \%$ of all cases, respectively. Of these patients, $59.1 \%$ had an NCI comorbidity score of 1 or 2 .

A total of 1767 patients received care from professionals in each discipline. After matching, no statistical differences were observed between patients receiving care from different professionals, in terms of sex, disease stage, comorbidities or adjuvant chemotherapy (table 1). Surgeons were slightly more likely than oncologists to change the treatment regimen $(\mathrm{p}=0.060)$. A greater proportion of patients received postoperative chemotherapy from low-volume surgeons than from low-volume oncologists (<25\%: $34.0 \%$ vs $14.3 \%$; $25 \%-50 \%$ : $31.9 \%$ vs $17.1 \%$, respectively, $\mathrm{p}<0.0001)$.

As shown in table 2, care paths did not have a significant influence on recurrence. Stage III colon cancer $(\mathrm{p}<0.0001)$, NCI score $3+(\mathrm{p}=0.029)$ and more cycles of adjuvant chemotherapy $(\mathrm{p}=0.027)$ were factors associated with a higher likelihood of disease recurrence. Patients who underwent changes in their chemotherapeutic agents had a higher recurrence rate than did patients who maintained the same regimen $(\mathrm{p}<0.0001)$.

We also conducted sensitivity analysis to examine the impact of treatment paths on recurrence among patients with stage II and stage III colon cancer (table 3). We observed no significant differences between the care paths in terms of recurrence. Changes in chemotherapy regimen were strongly associated with disease recurrence among patients in stage II (HR 5.97, p<0.0001) as well as those in stage III (HR 2.49, $\mathrm{p}<0.0001)$.

In terms of DFS, no statistical differences were observed in the outcomes of patients in stage II or stage III who followed different care paths (figure 2 and figure 3). The 5-year DFS rates in patients in stage II and stage 
Eligibility criteria:

1. Year of cancer diagnosis between 2005 and $2009(N=286,882)$

2. Colon cancer by site (ICD-O-3 $=\mathrm{C} 18)(N=25,005)$

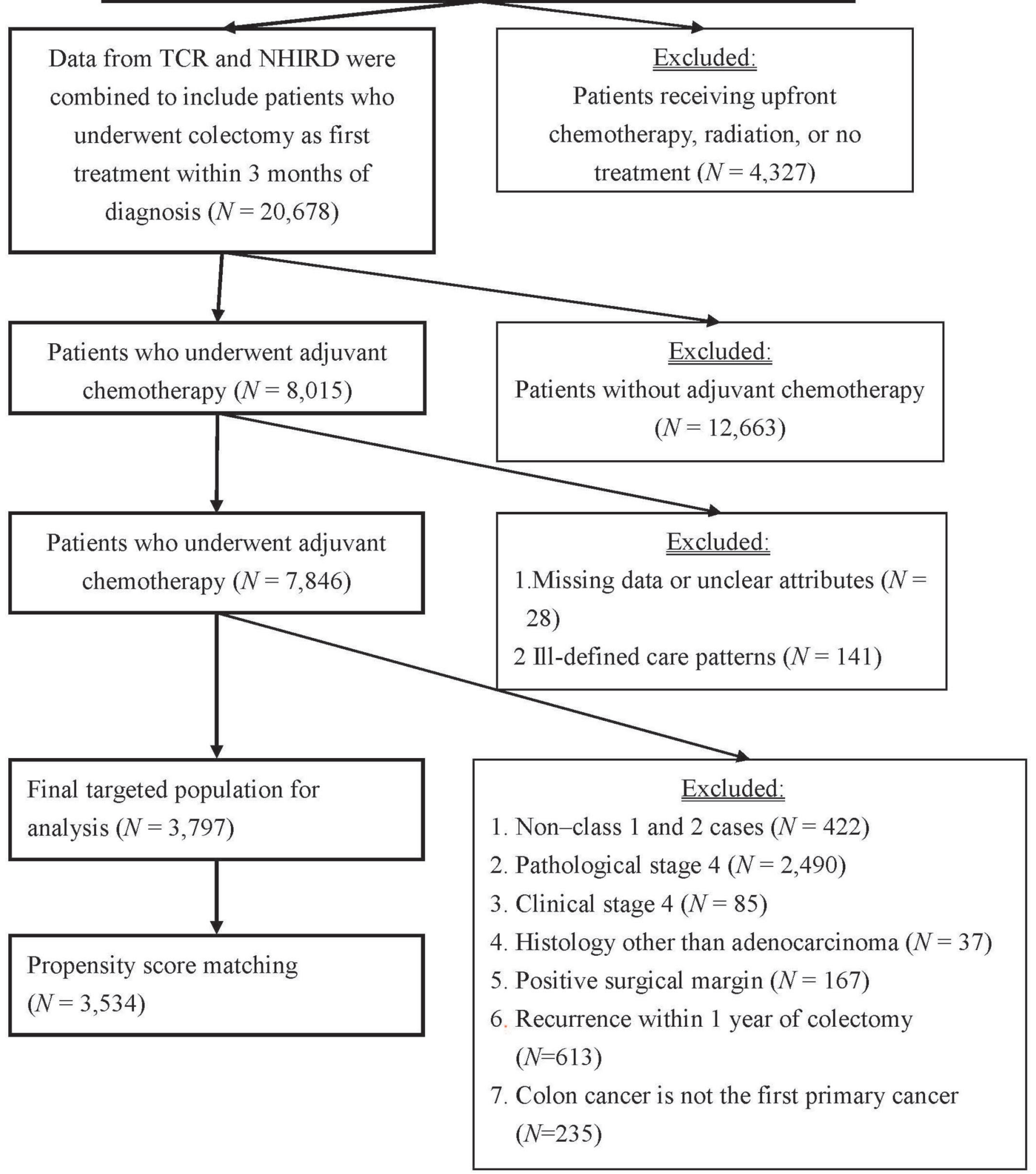

Figure 1 Flowchart of cohort selection from 2005 to 2009. NHIRD, National Health Insurance Research Database; TCR, Taiwan Cancer Registry. 
Table 1 Baseline characteristics of patients with colon cancer following different care paths

\begin{tabular}{|c|c|c|c|c|c|c|c|c|}
\hline & \multicolumn{4}{|c|}{ Before propensity score matching } & \multicolumn{4}{|c|}{ After propensity score matching* } \\
\hline & $\begin{array}{l}\text { Oncologist } \\
(n=1767) \\
N(\%)\end{array}$ & $\begin{array}{l}\text { Surgeon } \\
(n=2030) \\
N(\%)\end{array}$ & $\begin{array}{l}\text { Standardised } \\
\text { difference, \% }\end{array}$ & $P$ value & $\begin{array}{l}\text { Oncologist } \\
(n=1767) \\
N(\%)\end{array}$ & $\begin{array}{l}\text { Surgeon } \\
(n=1767) \\
N(\%)\end{array}$ & $\begin{array}{l}\text { Standardised } \\
\text { difference, \% }\end{array}$ & $P$ value \\
\hline Sex & & & & 0.12 & & & & 0.11 \\
\hline Male & 935 (52.9) & $1125(55.4)$ & 5.0 & & $935(52.9)$ & $982(55.6)$ & 5.3 & \\
\hline Mean (SD) & $59.14(12.4)$ & $59.76(12.7)$ & 4.9 & 0.13 & $59.14(12.4)$ & $59.99(12.5)$ & 4.9 & 0.04 \\
\hline$<50$ & $372(21.1)$ & $405(20.0)$ & 2.7 & & $372(21.1)$ & $337(19.1)$ & 4.9 & \\
\hline $50-60$ & $523(29.6)$ & $582(28.7)$ & 2.0 & & $523(29.6)$ & $516(29.2)$ & 0.9 & \\
\hline $61-70$ & $482(27.3)$ & $529(26.1)$ & 2.8 & & $482(27.3)$ & $461(26.1)$ & 2.7 & \\
\hline II & $481(27.2)$ & $493(24.3)$ & 6.7 & & $481(27.2)$ & $440(24.9)$ & 5.3 & \\
\hline III & $1262(71.4)$ & $1508(74.3)$ & 6.4 & & $1262(71.4)$ & $1302(73.7)$ & 5.1 & \\
\hline \multicolumn{2}{|c|}{$\mathrm{NCl}$ comorbidity score } & & & 0.84 & & & & 0.96 \\
\hline 0 & $523(29.6)$ & $564(27.8)$ & 4.0 & & $523(29.6)$ & $495(28.0)$ & 3.5 & \\
\hline 1 & $641(36.3)$ & $765(37.7)$ & 2.9 & & $641(36.3)$ & $668(37.8)$ & 3.2 & \\
\hline 2 & $376(21.3)$ & $472(23.3)$ & 4.7 & & $376(21.3)$ & $403(22.8)$ & 3.7 & \\
\hline $3+$ & $227(12.8)$ & $229(11.3)$ & 4.8 & & $227(12.8)$ & $201(11.4)$ & 4.5 & \\
\hline \multicolumn{9}{|c|}{ Adjuvant chemotherapy } \\
\hline \multicolumn{9}{|l|}{ No of cycles } \\
\hline Mean (SD) & $11.5(6.2)$ & $11.4(7.1)$ & 0.8 & 0.81 & $11.2(6.2)$ & $11.3(7.1)$ & 0.4 & 1.00 \\
\hline $51 \%-75 \%$ & $525(29.7)$ & $426(21.0)$ & 20.2 & & $525(29.7)$ & $370(20.9)$ & 20.3 & \\
\hline$>75 \%$ & $686(38.8)$ & $265(13.1)$ & 61.5 & & $686(38.8)$ & $233(13.2)$ & 61.1 & \\
\hline \multicolumn{9}{|l|}{ Recurrence } \\
\hline No & 1475 (83.5) & $1704(84.9)$ & & & 1475 (83.5) & $1491(84.4)$ & & \\
\hline Yes & $292(16.5)$ & $326(16.1)$ & & & $292(16.5)$ & $276(15.6)$ & & \\
\hline \multicolumn{9}{|c|}{ Disease-free survival, months } \\
\hline Mean (SD) & $46.91(19.2)$ & $47.43(19.2)$ & & & $46.91(19.2)$ & $47.65(19.1)$ & & \\
\hline Median (IQR) & $45(31-63)$ & 47 (31-63) & & & $45(31-63)$ & 47 (31-64) & & \\
\hline
\end{tabular}

The standardised differences of the 12 comorbid conditions were between 0.3 and 4.6 in unmatched data and between 0 and 4.5 in matched data (data not shown).

$P$ values were obtained using the Mantel-Haenszel test for categorical variables and generalised estimating equation regression for continuous variables.

*Variables used for propensity score matching included sex, age, stage, 12 comorbid conditions of $\mathrm{NCl}$ comorbidity index and history of regimen changes.

$\mathrm{NCl}$, National Cancer Institute.

III who received care from oncologists and surgeons were $90.02 \%$ versus $88.99 \%$ and $77.64 \%$ versus $79.99 \%$ ( $\mathrm{p}=0.628$ and $\mathrm{p}=0.137$, respectively). Patients with stage I colon cancer were excluded from analysis due to their favourable prognosis and relatively small sample size.

\section{DISCUSSION}

The number of cases of colon cancer newly diagnosed in Taiwan was 9584 in 2005 and 15140 in $2013 .{ }^{26}$ In the 1990s, adjuvant chemotherapy for colon cancer was shown to improve survival. ${ }^{172}$ At present, adjuvant 
Table 2 Disease recurrence categorised by clinical characteristics of patients, treatments, care paths and the case volume of primary care providers (Cox regression models)

\begin{tabular}{|c|c|c|c|c|c|c|}
\hline & \multicolumn{2}{|l|}{ Overall $(n=3534)$} & \multicolumn{2}{|c|}{ No change regimens $(n=3313)$} & \multicolumn{2}{|c|}{ Change regimens $(n=221)$} \\
\hline & HR $(95 \% \mathrm{Cl})$ & $P$ value & HR (95\% Cl) & $P$ value & HR $(95 \% \mathrm{Cl})$ & $P$ value \\
\hline \multicolumn{7}{|l|}{ Characteristics } \\
\hline \multicolumn{7}{|l|}{ Sex } \\
\hline Male & Ref & & Ref & & Ref & \\
\hline Female & 0.83 (0.70 to 0.98$)$ & 0.025 & 0.83 (0.69 to 0.99$)$ & 0.042 & 0.77 (0.47 to 1.26$)$ & 0.297 \\
\hline \multicolumn{7}{|l|}{ Age (years) } \\
\hline$<50$ & Ref & & Ref & & Ref & \\
\hline $50-60$ & 1.05 (0.83 to 1.34$)$ & 0.679 & 1.11 (0.85 to 1.44$)$ & 0.458 & 0.78 (0.42 to 1.45$)$ & 0.426 \\
\hline $61-70$ & $0.93(0.72$ to 1.20$)$ & 0.561 & 1.04 (0.79 to 1.37$)$ & 0.786 & 0.39 (0.18 to 0.84$)$ & 0.016 \\
\hline$>70$ & 0.96 (0.74 to 1.25$)$ & 0.741 & 0.99 (0.74 to 1.32$)$ & 0.943 & 0.94 (0.48 to 1.85$)$ & 0.862 \\
\hline \multicolumn{7}{|l|}{ Stage } \\
\hline 1 & 1.02 (0.41 to 2.53$)$ & 0.960 & 0.72 (0.23 to 2.27 ) & 0.569 & 2.32 (0.47 to 11.4$)$ & 0.299 \\
\hline II & Ref & & Ref & & Ref & \\
\hline III & 2.05 (1.63 to 2.59 ) & $<0.0001$ & 2.21 (1.73 to 2.82 ) & $<0.0001$ & 1.06 (0.53 to 2.13$)$ & 0.862 \\
\hline \multicolumn{7}{|l|}{$\mathrm{NCl}$ score } \\
\hline 0 & Ref & & Ref & & Ref & \\
\hline 1 & 1.06 (0.86 to 1.31$)$ & 0.570 & 0.97 (0.77 to 1.22$)$ & 0.780 & 1.77 (0.96 to 3.26$)$ & 0.067 \\
\hline 2 & 1.11 (0.87 to 1.41$)$ & 0.421 & 1.04 (0.80 to 1.34$)$ & 0.794 & 1.66 (0.78 to 3.53$)$ & 0.186 \\
\hline $3+$ & 1.37 (1.03 to 1.82$)$ & 0.029 & 1.25 (0.92 to 1.68$)$ & 0.150 & 2.89 (1.19 to 7.03$)$ & 0.019 \\
\hline \multicolumn{7}{|c|}{ Adjuvant chemotherapy } \\
\hline No of cycles & 1.01 (1. 00 to 1.03$)$ & 0.027 & 1.01 (1.00 to 1.02 ) & 0.133 & 1.03 (0.99 to 1.08$)$ & 0.101 \\
\hline \multicolumn{7}{|c|}{ Change in regimen } \\
\hline No & Ref & & - & & - & \\
\hline Yes & 2.75 (2.15 to 3.52 ) & $<0.0001$ & - & & - & \\
\hline \multicolumn{7}{|l|}{ Care paths } \\
\hline Oncologists & Ref & & Ref & & Ref & \\
\hline Surgeons & 0.88 (0.73 to 1.05$)$ & 0.148 & 0.87 (0.72 to 1.06$)$ & 0.165 & 0.94 (0.56 to 1.59$)$ & 0.820 \\
\hline \multicolumn{7}{|c|}{ Providers' case volume } \\
\hline$<25 \%$ & Ref & & Ref & & Ref & \\
\hline $25 \%-50 \%$ & 0.97 (0.77 to 1.23$)$ & 0.829 & 0.98 (0.76 to 1.26$)$ & 0.850 & 1.05 (0.55 to 2.00$)$ & 0.889 \\
\hline $51 \%-75 \%$ & 0.95 (0.74 to 1.21$)$ & 0.667 & 0.94 (0.72 to 1.21$)$ & 0.609 & 0.89 (0.44 to 1.80$)$ & 0.740 \\
\hline$>75 \%$ & 0.94 (0.73 to 1.21$)$ & 0.634 & 0.89 (0.68 to 1.16$)$ & 0.381 & 1.66 (0.80 to 3.45$)$ & 0.175 \\
\hline
\end{tabular}

$\mathrm{NCl}$, National Cancer Institute.

chemotherapy for high-risk patients with stage II and stage III colon cancer is the standard. Surgeons in Taiwan typically prescribed adjuvant treatment themselves or refer the patient to an oncologist.

To the best of our knowledge, this is the first study to investigate whether the care paths implemented by oncologists and non-oncologists differ in terms of patient outcomes. We observed no difference in DFS despite differences in the care paths (figures 2 and 3). A similar result was observed in two early retrospective cohort studies that compared patient outcomes among different disciplines. The study by Silber $e t$ al applied a research design similar to that of the current study. They hypothesised that patients would benefit more (in terms of postoperative survival) when receiving chemotherapy from a medical oncologist rather than a gynaecologic oncologist. Nonetheless, the two groups of patients presented equal survival results. They explained that their results could perhaps be attributed to the imperfect measurement of chemotherapy and the assignment of providers. ${ }^{28}$ Earle reported that after adjusting for surgeon types and patient characteristics, gynaecologic oncologists and general gynaecologists achieved outcomes that were marginally superior to those of general surgeons. However, the details and jurisdiction of specialists in chemotherapy were not discussed. In addition, characterising chemotherapy using a variable 
Table 3 Disease recurrence in patients with stage II or III colon cancer, categorised according to clinical characteristics, treatments, care paths and case volume of primary care providers

\begin{tabular}{|c|c|c|c|c|}
\hline & \multicolumn{2}{|l|}{ Stage II $(n=921)$} & \multicolumn{2}{|l|}{ Stage III $(n=2564)$} \\
\hline & HR $(95 \% \mathrm{Cl})$ & $P$ value & HR $(95 \%$ Cl) & $P$ value \\
\hline \multicolumn{5}{|l|}{ Characteristics } \\
\hline \multicolumn{5}{|l|}{ Sex } \\
\hline Male & Ref & & Ref & \\
\hline Female & 1.17 (0.76 to 1.79$)$ & 0.484 & 0.78 (0.65 to 0.94$)$ & 0.009 \\
\hline \multicolumn{5}{|l|}{ Age (years) } \\
\hline$<50$ & Ref & & Ref & \\
\hline $50-60$ & 1.08 (0.57 to 2.05$)$ & 0.806 & 1.00 (0.77 to 1.30$)$ & 0.997 \\
\hline $61-70$ & 1.34 (0.71 to 2.55$)$ & 0.370 & 0.86 (0.65 to 1.13$)$ & 0.267 \\
\hline$>70$ & 1.43 (0.72 to 2.83$)$ & 0.309 & 0.88 (0.66 to 1.17$)$ & 0.384 \\
\hline \multicolumn{5}{|l|}{$\mathrm{NCl}$ score } \\
\hline 0 & Ref & & Ref & \\
\hline 1 & 1.12 (0.64 to 1.94$)$ & 0.693 & 1.05 (0.84 to 1.32$)$ & 0.670 \\
\hline 2 & 1.29 (0.68 to 2.43 ) & 0.441 & 1.10 (0.85 to 1.44$)$ & 0.466 \\
\hline $3+$ & 1.61 (0.78 to 3.33) & 0.198 & 1.32 (0.97 to 1.80$)$ & 0.083 \\
\hline \multicolumn{5}{|c|}{ Adjuvant chemotherapy } \\
\hline No of cycles & 1.01 (0.98 to 1.04$)$ & 0.509 & 1.02 (1.00 to 1.03$)$ & 0.035 \\
\hline \multicolumn{5}{|c|}{ Change in regimen } \\
\hline No & Ref & & Ref & \\
\hline Yes & 5.97 (2.98 to 11.97$)$ & $<0.0001$ & 2.49 (1.90 to 3.26$)$ & $<0.0001$ \\
\hline \multicolumn{5}{|l|}{ Care paths } \\
\hline Oncologists & Ref & & Ref & \\
\hline Surgeons & 1.00 (0.65 to 1.55$)$ & 0.986 & 0.85 (0.69 to 1.03 ) & 0.102 \\
\hline \multicolumn{5}{|c|}{ Providers' case volume } \\
\hline$<25 \%$ & Ref & & Ref & \\
\hline $25 \%-50 \%$ & 1.32 (0.74 to 2.32$)$ & 0.346 & 0.94 (0.73 to 1.23 ) & 0.662 \\
\hline $51 \%-75 \%$ & $0.48(0.24$ to 0.96$)$ & 0.038 & 1.03 (0.79 to 1.34$)$ & 0.854 \\
\hline$>75 \%$ & 1.16 (0.65 to 2.05$)$ & 0.613 & 0.92 (0.69 to 1.22$)$ & 0.554 \\
\hline
\end{tabular}

$\mathrm{NCl}$, National Cancer Institute.

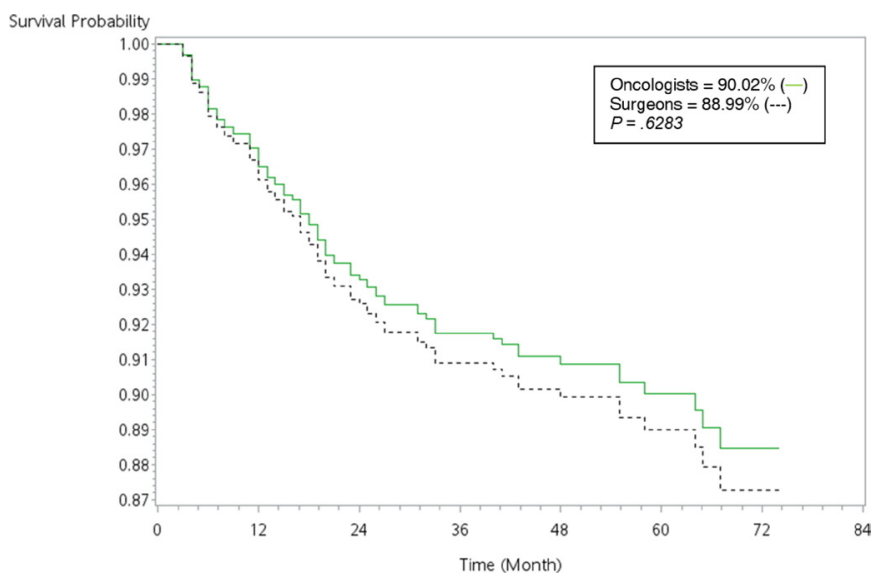

Figure 2 Disease-free survival of patients with stage II cancer by different care paths.

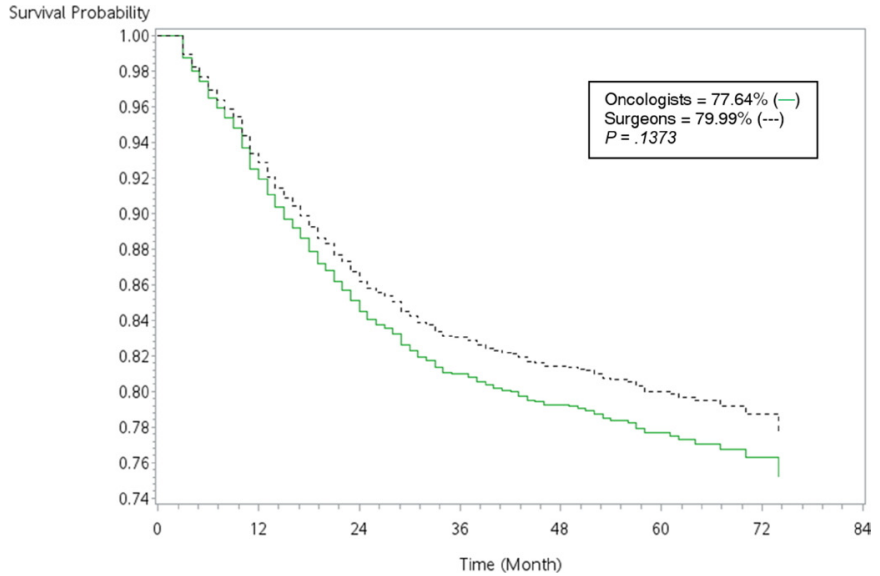

Figure 3 Disease-free survival of patients with stage III cancer by different care paths. 
of all-or-none activity identified in billing claims tends towards oversimplification and can be detrimental to overall findings. ${ }^{29}$ Compared with the designs used in the aforementioned studies, the present study included more details pertaining to the administration of chemotherapy.

Our results indicate that changes in regimen or dose are significantly associated with disease recurrence. Patients who were prescribed chemotherapy by surgeons underwent more regimen changes than did those who were prescribed chemotherapy by oncologists. Nonetheless, the survival of patients who were under the care of surgeons was no worse, as one may intuitively infer. Nonetheless, our study design cannot be used to determine whether surgeons made more change regimens or to characterise the outcomes in cases where changes were made. We included regimen changes in our statistical models to control for confounding factors related to regimen changes, which are not necessarily observable in our data. We also conducted sensitivity analysis that included only patients who had not undergone regimen changes (table 2). Our results revealed no statistical differences in disease recurrence among patients treated by oncologists or surgeons. The actual implications of these findings remain unclear due to the fact that clinical information of the patients and details of the chemotherapy they received are unknown.

We also determined that the two paths were very similar in terms of the number of chemotherapy cycles (mean: 11.2 vs $11.3, \mathrm{p}=1.00)$. This differs from the report by Silber et al, in which it was reported that medical oncologists prescribed chemotherapy for longer durations than did gynaecologic oncologists. Anecdotal evidence suggests that medical oncologists may be referred a larger proportion of patients with a refractory disease requiring more courses of salvage treatment. A wider range of cycles may stem from the various regimens employed under various treatment paradigms. We employed the unit of weeks from the billing data, which is less satisfactory than the unit of real cycles. Nonetheless, neither Silber et al nor we could verify the assumption that chemotherapy prescribed through dissimilar in-house logistics affects survival.

We found that the case volume of patients managed by the health provider was not associated with disease recurrence. This contradicts the findings of previous studies, which reported a positive association between the volume of patients and colorectal cancer outcomes. ${ }^{30}{ }^{31}$ However, colorectal surgery is a low-risk procedure, such that the incidence of incomplete surgical staging is lower than that observed in gynaecological malignancies. ${ }^{32}$ The results in most previous studies were in terms of short-term postoperative mortality and length of stay or costs. In those studies, adjuvant chemotherapy was seldom discussed ${ }^{33}$ It was not possible to compare most of the studies directly due to differences in volume definitions and outcome measures. ${ }^{34} 35 \mathrm{In}$ this study, the provider's patient volume was not associated with recurrence; however, we found that $65.9 \%$ of patients who received chemotherapy from surgeons were treated by professionals with low case volumes $(\leq 50 \%)$. Conversely, we found that $68.5 \%$ of patients who received chemotherapy from oncologists were treated by professionals with high case volumes $(>50 \%)$. One previous study reported a $5 \%$ improvement in survival for every additional patient shared between surgeons and oncologists. ${ }^{2}$ However, these findings seem to imply a certain degree of spillover. When surgeons reached the maximum number of patients they could treat, some patients were referred to or voluntarily went to oncologists. One study on ovarian cancer reported that a surgeon's volume of patients is not predictive of survival; however, a referral to a medical oncologist (or lack thereof) was a strong predictor. ${ }^{36}$ The reasons for referring or not referring patients to oncologists remain to be investigated, particularly within a fee-for-service payment system. ${ }^{37}$

Previous studies have shown that adherence to clinical guidelines in the administration of cancer treatment would have the same effect on survival, regardless of the specialty of the practitioner. ${ }^{30}{ }^{38}$ Nonetheless, adherence to clinical guidelines could be expected to promote the trespassing of professional boundaries. Boundary blurring can be affected by any number of factors, such as culture, financial and non-financial incentives, the scope of work, knowledge and skills, role and identity, and power status. ${ }^{73739-41}$ Collaboration between disciplines has numerous benefits in terms of patient outcomes. Nonetheless, in Taiwan, the status of medical oncologists has been devalued despite international recognition of their contributions. ${ }^{42}$

This study has a number of limitations. First, randomised controlled trials are the most reliable means of obtaining evidence in the field of medicine; however, conducting a prospective randomised trial to compare the outcomes of patients undergoing different care paths would be impossible. Furthermore, cross-boundary work is not a major concern in healthcare systems outside Taiwan. The only related retrospective studies have focused on the management of ovarian cancer, which has for decades involved a power struggle in the American Gynecology Society. ${ }^{5}{ }^{43}$ Second, we were unable to identify other influential factors, such as variations in dose intensity, the number of cycles across providers, the preferences of patients, the physical frailty of patients or treatment complications. To mitigate the potential confounding effects of changes in the treatment regimen, we performed sensitivity analysis in which 221 patients who underwent regimen changes were excluded. The results were no different than those obtained using the entire sample. The dataset was the factor limiting our definitions of regimen changes. In the future, researchers should make an effort to take these unobserved factors into account. Third, we focused exclusively on patients who received adjuvant chemotherapy, based on strict eligibility criteria. This resulted in the exclusion of a substantial number of patients who underwent paths other than those involving surgeons or oncologists as well as those who experienced recurrence within 1 year. 


\section{CONCLUSIONS}

The prescribing of systemic chemotherapy by non-oncologists is a common practice in the single-payer global healthcare system in Taiwan. This is the first study to address the fundamental question of whether the discipline of the care provider affects patient outcomes. Our analysis does not favour any path of care and our findings indicate no difference in patient survival, regardless of who oversaw the administration of chemotherapy. Nonetheless, one must not jump to any conclusions at this point with regard to the blurring of professionalism boundaries. Moreover, these findings are not applicable to other malignancies or other disease stages. Further study using outcome measures other than survival time should be conducted in the future.

\section{Author affiliations}

${ }^{1}$ Division of Hematology and Oncology, Department of Internal Medicine, Taipei Medical University Hospital, Taipei, Taiwan

${ }^{2}$ Division of Hematology and Oncology, Department of Internal Medicine, School of Medicine, College of Medicine, Taipei Medical University, Taipei, Taiwan ${ }^{3}$ Institute of Health Policy and Management, College of Public Health, National Taiwan University, Taipei, Taiwan

${ }^{4}$ Innovation and Policy Center for Population Health and Sustainable Environment, College of Public Health, National Taiwan University, Taipei, Taiwan

${ }^{5}$ Department of Health Care Management, National Taipei University of Nursing and Health Sciences, Taipei, Taiwan

Contributors Conceptualisation: C-IH, RN-CK. Data curation: C-CL, RN-CK. Formal analysis: C-CL, H-YT. Funding acquisition: KP-C. Investigation: C-IH. Methodology: C-IH, RN-CK. Resources: RN-CK, KP-C. Software: C-CL, H-YT. Supervision: RN-CK. Validation: RN-CK, C-CL, H-YT. Writing-original draft: C-IH. Writing-review and editing: C-IH, RN-CK.

Funding We acknowledge the support from the Ministry of Science and Technology (MOST), Taiwan under grant no. 102-2410-H-002-123-SS3.

Competing interests None declared.

Patient consent for publication Not required.

Ethics approval The protocol for this study was approved by the institutional review board of National Taiwan University (protocol no. 201501053RINA).

Provenance and peer review Not commissioned; externally peer reviewed.

Data sharing statement Additional unpublished data are not publicly available. The authors are authorised to use the data in this study; however, we do not own the datasets and cannot prevent access to them. All requests for data access must be approved by the Department of Statistics, Ministry of Health and Welfare (Taiwan). Researchers who are interested in the data may also apply to the Department of Statistics, Ministry of Health and Welfare (Taiwan) by contacting Ms Wu Tzu-Hui (stcarolwu@mohw.gov.tw) or Mr Zongying Lin (st-zylin@mohw.gov.tw).

Open access This is an open access article distributed in accordance with the Creative Commons Attribution Non Commercial (CC BY-NC 4.0) license, which permits others to distribute, remix, adapt, build upon this work non-commercially, and license their derivative works on different terms, provided the original work is properly cited, appropriate credit is given, any changes made indicated, and the use is non-commercial. See: http://creativecommons.org/licenses/by-nc/4.0/.

\section{REFERENCES}

1. Oeffinger KC, McCabe MS. Models for delivering survivorship care. $J$ Clin Oncol 2006;24:5117-24.

2. Hussain T, Chang HY, Veenstra CM, et al. Collaboration between surgeons and medical oncologists and outcomes for patients with stage III colon cancer. J Oncol Pract 2015;11:e388-97.

3. Steven Allender PS, Peto V, Rayner M, et al. Ramon LuengoFernandez and Alastair Gray. European cardiovascular disease statistics, 2008.
4. Wilson CT, Fisher ES, Welch HG, et al. U.S. Trends in CABG hospital volume: the effect of adding cardiac surgery programs. Health Aff 2007;26:162-8.

5. Zetka JR. Establishing specialty jurisdictions in medicine: the case of American obstetrics and gynaecology. Sociol Health IIIn 2011;33:837-52.

6. Winchester DP. What's best for the patient? Am J Surg 2007;194:278.

7. Nancarrow SA, Borthwick AM. Dynamic professional boundaries in the healthcare workforce. Sociol Health IIIn 2005;27:897-919.

8. Siminoff LA, Zhang A, Saunders Sturm CM, et al. Referral of breast cancer patients to medical oncologists after initial surgical management. Med Care 2000;38:696-704.

9. Krueger G, Canellos G. Where does hematology end and oncology begin? Questions of professional boundaries and medical authority. $J$ Clin Oncol 2006;24:2583-8.

10. Weiss MC, Sutton J. The changing nature of prescribing: pharmacists as prescribers and challenges to medical dominance. Sociol Health IIIn 2009;31:406-21.

11. Gilbert L. To diagnose, prescribe and dispense: whose right is it? the ongoing struggle between pharmacy and medicine in South Africa. Current Sociology 2001;49:97-118.

12. Davidoff AJ, Rapp T, Onukwugha E, et al. Trends in disparities in receipt of adjuvant therapy for elderly stage III colon cancer patients: the role of the medical oncologist evaluation. Med Care 2009;47:1229-36.

13. Leonard RC, Miles D, Thomas R, et al. UK Breast Cancer Neutropenia Audit Group. Impact of neutropenia on delivering planned adjuvant chemotherapy: UK audit of primary breast cancer patients. Br J Cancer 2003;89:2062-8.

14. Lyman $\mathrm{GH}$. Chemotherapy dose intensity and quality cancer care. Oncology 2006;20(14 Suppl 9):16-25

15. Lyman GH. Impact of chemotherapy dose intensity on cancer patient outcomes. J Natl Compr Canc Netw 2009;7:99-108.

16. Dobie SA, Baldwin LM, Dominitz JA, et al. Completion of therapy by Medicare patients with stage III colon cancer. J Natl Cancer Inst 2006;98:610-9.

17. Wolmark N, Rockette H, Mamounas E, et al. Clinical trial to assess the relative efficacy of fluorouracil and leucovorin, fluorouracil and levamisole, and fluorouracil, leucovorin, and levamisole in patients with Dukes' B and C carcinoma of the colon: results from National Surgical Adjuvant Breast and Bowel Project C-04. J Clin Oncol 1999;17:3553-9.

18. Haller DG, Catalano PJ, Macdonald JS, et al. Phase III study of fluorouracil, leucovorin, and levamisole in high-risk stage II and III colon cancer: final report of Intergroup 0089. J Clin Oncol 2005;23:8671-8.

19. O'Connell MJ, Mailliard JA, Kahn MJ, et al. Controlled trial of fluorouracil and low-dose leucovorin given for 6 months as postoperative adjuvant therapy for colon cancer. $J$ Clin Oncol 1997;15:246-50.

20. Marshall JL, Haller DG, de Gramont A, et al. Adjuvant therapy for stage II and III colon cancer: consensus report of the international Society of Gastrointestinal Oncology. Gastrointest Cancer Res 2007;1:146-54

21. André T, Boni $C$, Navarro $M$, et al. Improved overall survival with oxaliplatin, fluorouracil, and leucovorin as adjuvant treatment in stage II or III colon cancer in the MOSAIC trial. J Clin Oncol 2009;27:3109-16.

22. André $\mathrm{T}$, Boni $\mathrm{C}$, Mounedji-Boudiaf L, et al. Multicenter International Study of Oxaliplatin/5-Fluorouracil/Leucovorin in the Adjuvant Treatment of Colon Cancer (MOSAIC) Investigators. Oxaliplatin, fluorouracil, and leucovorin as adjuvant treatment for colon cancer. $N$ Engl J Med 2004;350:2343-51.

23. Klabunde CN, Legler JM, Warren JL, et al. A refined comorbidity measurement algorithm for claims-based studies of breast, prostate, colorectal, and lung cancer patients. Ann Epidemiol 2007;17:584-90.

24. Tsai HY, Chung KP, Kuo RN. Impact of targeted therapy on the quality of end-of-life care for patients with non-small-cell lung cancer: a population-based study in Taiwan. J Pain Symptom Manage 2018;55:798-807.

25. Yeh YC, Liu CJ, Kuo RN, et al. Association of adjuvant antiviral therapy with risk of cancer progression and deaths in patients with hepatitis-B-virus-related hepatocellular carcinoma following curative treatment: a nationwide cohort study. PLoS One 2014;9:e102051.

26. Welfare MoHa. Health promotion adinistration. https:// wwwhpagovtw/EngPages/Indexaspx (assessed May 2017).

27. Moertel CG, Fleming TR, Macdonald JS, et al. Intergroup study of fluorouracil plus levamisole as adjuvant therapy for stage II/Dukes' B2 colon cancer. J Clin Oncol 1995;13:2936-43. 
28. Silber JH, Rosenbaum PR, Polsky D, et al. Does ovarian cancer treatment and survival differ by the specialty providing chemotherapy? J Clin Oncol 2007;25:1169-75.

29. Earle CC, Schrag D, Neville BA, et al. Effect of surgeon specialty on processes of care and outcomes for ovarian cancer patients. $J$ Natl Cancer Inst 2006;98:172-80.

30. Seagle B-LL, Strohl AE, Dandapani M, et al. Survival disparities by hospital volume among American women with gynecologic cancers. JCO Clin Cancer Inform 2017;1:1-15.

31. Archampong D, Borowski D, Wille-Jørgensen P, et al. Workload and surgeon's specialty for outcome after colorectal cancer surgery. Cochrane Database Syst Rev 2012;3:CD005391.

32. Young RC, Decker DG, Wharton JT, et al. Staging laparotomy in early ovarian cancer. JAMA 1983;250:3072-6.

33. Hillner BE, Smith TJ, Desch CE. Hospital and physician volume or specialization and outcomes in cancer treatment: importance in quality of cancer care. J Clin Oncol 2000;18:2327-40.

34. Kvåle K, Bondevik M. Patients' perceptions of the importance of nurses' knowledge about cancer and its treatment for quality nursing care. Oncol Nurs Forum 2010;37:436-42.

35. Mohan S, Wilkes LM, Ogunsiji O, et al. Caring for patients with cancer in non-specialist wards: the nurse experience. Eur J Cancer Care 2005;14:256-63.
36. Woodman C, Baghdady A, Collins S, et al. What changes in the organisation of cancer services will improve the outcome for women with ovarian cancer? Br J Obstet Gynaecol 1997;104:135-9.

37. Saver RS. Is it really all about the money? Reconsidering nonfinancial interests in medical research. The Journal of Law, Medicine \& Ethics 2012:40:467-81.

38. Erickson BK, Martin JY, Shah MM, et al. Reasons for failure to deliver National Comprehensive Cancer Network (NCCN)-adherent care in the treatment of epithelial ovarian cancer at an NCCN cancer center. Gynecol Oncol 2014;133:142-6.

39. Kroezen M, van Dijk L, Groenewegen PP, et al. Knowledge claims, jurisdictional control and professional status: the case of nurse prescribing. PLoS One 2013;8:e77279.

40. Hall P. Interprofessional teamwork: professional cultures as barriers. $J$ Interprof Care 2005;19(Supp 1):188-96.

41. Baxter SK, Brumfitt SM. Professional differences in interprofessional working. J Interprof Care 2008;22:239-51.

42. Casali PG. Medical oncology: the long-awaited prize of recognition. Ann Oncol 2011;22:1695-7.

43. Kehoe S, Powell J, Wilson S, et al. The influence of the operating surgeon's specialisation on patient survival in ovarian carcinoma. $\mathrm{Br}$ J Cancer 1994;70:1014-7. 